\title{
Congenital syphilis: not gone and all too forgotten
}

\author{
Mary L. Kamb \\ Atlanta, USA
}

$\mathrm{T}$ This issue features a retrospective study of clinical records from one health facility in rural Tanzania in which the investigators uncovered an unexpected number of congenital syphilis cases among infants (Page 125-131). The authors point out that congenital syphilis is an age-old disease that has fallen off the priority list of the global health agenda, yet continues to cause substantial morbidity in countries with limited resources. They further note that syphilis can be cheaply and easily cured with penicillin anywhere in the world, supporting that the most important reason that this infection continues to kill and maim infants is through simple neglect.

The evidence supports that the authors' assertions are correct. The World Health Orgnization (WHO) estimates that each year between 715000 and 1575000 pregnant women are infected with syphilis, and most of them (up to $80 \%$ ) will suffer a serious adverse pregnancy outcome. ${ }^{[1]}$ Among live-born infants of infected mothers, about half go on to have congenital syphilis infection as depicted in this article; but these surviving children represent just the tip of the iceberg. In low income settings, syphilis is the most common infection associated with fetal loss or stillbirth, occurring in up to $40 \%$ of pregnancies among infected women who are inadequately treated. ${ }^{[1-3]}$ Syphilis infection also contributes importantly to preterm delivery, affecting $20 \%-33 \%$ of infants born to untreated mothers, and through this means cause a substantial source of early neonatal death. ${ }^{[1,3]}$ Morbidity aside, the global perinatal mortality associated with syphilis is estimated at about 327000 cases each year-similar to or exceeding the perinatal deaths estimated for HIV, malaria or tetanusand almost all these cases could be prevented. ${ }^{[4]}$

Adverse pregnancy outcomes caused by maternal syphilis infection are avoided through screening early

Author Affiliations: Division of STD Prevention, U. S. Centers for Disease Control and Prevention (CDC), Atlanta, Georgia, USA (Kamb ML)

Corresponding Author: Mary L. Kamb, MD, MPH, Division of STD Prevention, U. S. Centers for Disease Control and Prevention (CDC), Atlanta, Georgia, USA (Email: mlk5@cdc.gov)

doi:10.1007/s12519-010-0024-3

(C)Children's Hospital, Zhejiang University School of Medicine, China and Springer-Verlag Berlin Heidelberg 2010. All rights reserved. in pregnancy (preferably at the first antenatal visit) with prompt treatment for women with positive tests. This intervention is already recommended by the WHO and United Nations Children's Fund as part of a basic antenatal clinic (ANC) care package for maternal and newborn child health $(\mathrm{MNCH})$ services. ${ }^{[5-7]}$ In fact, universal syphilis screening for pregnant women is already recommended in the national reproductive health strategies of almost every nation of the world. ${ }^{[1,8]}$ As a public health intervention, maternal syphilis screening is recognized as among the most cost-effective interventions in existence, estimated to cost just $\$ 7$ to $\$ 10$ per disability-adjusted life year (DALY) saved in medium to high prevalence settings. ${ }^{[1]}$ And yet syphilis screening during pregnancy still does not occur in many low and middle income countries. Why not?

Historically, there have been many reasons why pregnant women are still not routinely tested and treated for syphilis. One is lack of health care access: Globally, a quarter of pregnant women still receive no antenatal care, and this proportion tends to be higher in countries, such as Tanzania, with higher syphilis prevalence. Another is limited local resources. Many health districts have insufficient funds to provide syphilis testing free of charge, and women often lack the personal resources to pay for the testing out of pocket. Another is lack of sufficient public health infrastructure. The RPR test that is classically used to screen for syphilis is relatively inexpensive; but it requires sufficient basic laboratory capacity to be done correctly. Where ANC clinics lack basic laboratory capacity, tests must be sent on to higher level health facilities and women must wait for results. This is a sure recipe for loss of women to follow up treatment in settings in which it is difficult for women to reach ANC care in the first place.

In fact, many of these factors are being addressed. The Millennium Development Goals' targets for women and children's health have emphasized health care access, and ANC attendance is increasing even in the world's poorest nations. Syphilis testing options have changed greatly over the past two decades. Through the STI Diagnostics Initiative (SDI), part of the Special Program for Research and Training in Tropical Disease (TDR), a number of rapid point-of-care treponemal tests that are easy to use and heat stable have been developed, field tested and made available to health ministries at low cost through a WHO bulk purchasing mechanism. ${ }^{[9]}$ These rapid tests allow women to be 
screened for syphilis exposure at their primary care visit and, if positive, treated on the spot at a cost of $\$ 0.19-\$ 1.00$ per test. The availability of these simple tests, coupled with the low cost of a single penicillin injection (recommended treatment to prevent congenital syphilis), means that it is now possible for any woman attending ANC care, no matter how remote the setting, be tested and promptly treated for syphilis as part of basic health services. In addition to these factors, national programs are increasingly using systems that link interventions, such as testing for syphilis and HIV prevention of mother-to-child transmission, to promote efficiencies and better quality health services. ${ }^{[10]}$

But despite this progress, something is still lacking. As our Tanzanian colleagues point out, perhaps the most important reason that syphilis continues to cause unnecessary perinatal mortality may lie with us, the health care providers and public health policy makers: Many of us are simply unaware of the terrible toll that syphilis takes on pregnancies, or that syphilis remains a relatively common disease. Many of us are unaware of the technical advances in testing, and the very low costs involved. If we fully understood the true burden of syphilis-associated pregnancy and the options that are already available to us, would we not mount the political will to stop this public health failure?

In October 2007 at the Women Deliver Conference in London, the WHO launched a new global initiative for the "Elimination of Congenital Syphilis as a Public Health Problem", with signatories from more than 60 nations, international health agencies and academic institutions around the world. The initiative seeks to bring congenital syphilis back on the public health agenda while promoting elimination as a means of supporting basic reproductive health services rather than a disease-specific program. In this way the effort can help bolster overall MNCH systems and improve maternal and infant health outcomes, even above and beyond those caused by syphilis. While little funding for the initiative exists to date, in fact limited funding is needed: with syphilis screening and treatment costing less than $\$ 1$ per woman served, these funds can and should be identified.

We are closer than ever, but finally eliminating congenital syphilis requires our continued diligence and advocacy. We need to join together to increase awareness among policy makers that syphilis continues to silently destroy pregnancies, and that the means of preventing its complications are now available and affordable even for poor nations. We need commitment by health providers to implement syphilis testing and prompt treatment of positives as part of the basic antenatal package provided for all pregnant womenuniversally - and not simply to those who can afford to pay. We need public health dedication by public health program managers to ensure comprehensive provider trainings, quality assurance strategies and routine monitoring systems link congenital syphilis and other proven-effective interventions into routine ANC services. And we need creative thinking in the global public health community on how to promote unified health systems that include cost-effective interventions, like maternal syphilis screening and treatment, as part of existing local health programs. Approached in this way, our congenital syphilis elimination efforts can both slash the toll of syphilis on pregnancies and promote improved maternal and infant health.

Funding: None.

Ethical approval: Not needed.

Competing interest: None.

Contributors: Kamb ML is the single author of this paper.

\section{References}

1 World Health Organization. The Global Elimination of Congenital Syphilis: Rationale and Strategy for Action. WHO, 2007. NLM classification: WC 161. Available at: www.who.int/ reproductivehealth/publications/rtis/9789241595858/en/index. html.

2 Watson-Jones D, Changalucha J, Gumodoka B, Weiss H, Rusizoka M, Ndeki L, et al. Syphilis in pregnancy in Tanzania. I. Impact of maternal syphilis on outcome of pregnancy. J Infect Dis 2002;186:940-947.

3 Goldenberg RL, Thompson C. The infectious origins of stillbirth. Am J Obstet Gynecol 2003;189:861-873.

4 Schmid GP, Stoner BP, Hawkes S, Broutet N. The need and plan for global elimination of congenital syphilis. Sex Transm Dis 2007;34(7 Suppl):S2-4.

5 World Health Organization. Integrating STI/RTI Care for Reproductive Health. Sexually transmitted and other reproductive tract infections: a guide to essential practice. WHO, 2005. NLM classification: WC 140. Available at: http://www.who.int/ reproductive-health.

6 World Health Organization. Department of Making Pregnancy Safer. Standards for maternal and neonatal care. WHO, 2007. Available at: http://www.who.int/making_pregnancy_safer/ documents.

7 UNICEF. The State of the World's Children 2009: Maternal and Newborn Health. UNICEF, 2009. Available at: http://www. unicef.org/publications/index_47127.html.

8 Hossain M, Broutet N, Hawkes S. The elimination of congenital syphilis: a comparison of the proposed World Health Organization action plan for the elimination of congenital syphilis with existing national maternal and congenital syphilis policies. Sex Transm Dis 2007;34(7 Suppl):S22-30.

9 Peeling RW, Ye H. Diagnostic tools for preventing and managing maternal and congenital syphilis: an overview. Bull World Health Organ 2004;82:439-446.

10 Peeling RW, Mabey D, Fitzgerald DW, Watson-Jones D. Avoiding HIV and dying of syphilis. Lancet 2004;364:1561-1563.

Received October 7, 2009

Accepted after revision October 30, 2009 